\title{
Novel System Inversion Algorithm With Application to Oversampled Perfect Reconstruction Filter Banks
}

\author{
Sander Wahls, Student Member, IEEE, and Holger Boche, Senior Member, IEEE
}

\begin{abstract}
We derive a novel algorithm for linear (discrete-time) system inversion with decision delay and frequency weighted $\mathcal{H}_{2}$ norm criterion. Complexity of the algorithm grows only linearly with the decision delay. The algorithm also applies to certain singular cases where optimal inverses may be nonunique. In that case, the set of optimal inverses is parametrized. A Scilab implementation of the algorithm is provided. Applications in oversampled perfect reconstruction filter banks are given.
\end{abstract}

Index Terms-Filter bank, IIR digital filters, inversion, MIMO systems, perfect reconstruction, state space methods.

\section{NOTATION}

Notation in this paper is similar to [1]. In particular, $\mathcal{R H}_{\infty}^{q \times p}$ denotes the space of (discrete-time) stable and causal rational $q \times p$ matrices. We often drop the dimensions when possible. For any $\Psi \in \mathcal{R} \mathcal{H}_{\infty}$, we define the para-Hermitian $\Psi^{\sim}(z):=\Psi\left(\bar{z}^{-1}\right)^{*}$ and the $\mathcal{H}_{2}$ norm $\|\Psi\|_{2}^{2}:=\int_{\theta=0}^{2 \pi} \operatorname{trace}\left\{\Psi^{\sim}\left(e^{i \theta}\right) \Psi\left(e^{i \theta}\right)\right\}(d \theta) /(2 \pi)$. If $\Psi$ is tall and has full normal rank, we further set $\Psi^{\dagger}:=\left(H^{\sim} H\right)^{-1} H^{\sim}$. The Moore-Penrose pseudoinverse of any $\Phi \in \mathbb{C}^{q \times p}$ is denoted by $\Phi^{+}$.

\section{INTRODUCTION}

$\mathbf{S}$ TABLE inversion of rational matrices is a commonly encountered problem in filter bank theory, which arises when filter banks are described via polyphase representation [2], [3], [4, Ch. 5.5]. The polyphase representation of a typical analysis-synthesis filter bank cascade is depicted in Fig. 1. The rational matrices $H(z)$ and $G(z)$ are called the (polyphase matrices of the) analysis and synthesis filter bank, respectively. The filter bank cascade is called oversampled if $H(z)$ is a tall matrix, or, equivalently, $G(z)$ is wide. When $G(z)$ is a (delayed) left-inverse of $H(z)$, i.e., $G(z) H(z)=z^{-L} I$ for some decision delay $L \geq 0$, the cascade is said to achieve perfect reconstruction (PR). A typical task arising in many applications is the following. Given an analysis filter bank $H(z)$, find the optimal synthesis filter bank $G(z)$ that leads to PR. The computation

Manuscript received March 30, 2009; accepted February 22, 2010. Date of publication March 01, 2010; current version published May 14, 2010. The associate editor coordinating the review of this manuscript and approving it for publication was Dr. Anna Scaglione. This work has been supported by the German Research Foundation (DFG) under Grant BO 1734/11-2.

The authors are with Technische Universität Berlin, Lehrstuhl für Informationstheorie und Theoretische Informationstechnik, Werner-von-Siemens-Bau (HFT 6), 10587 Berlin, Germany (e-mail: sander.wahls@mk.tu-berlin.de; holger.boche@mk.tu-berlin.de).

Color versions of one or more of the figures in this paper are available online at http://ieeexplore.ieee.org.

Digital Object Identifier 10.1109/TSP.2010.2044606

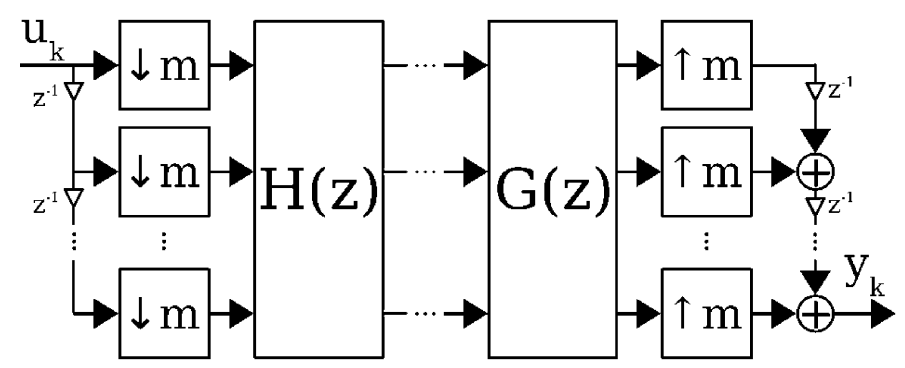

Fig. 1. Analysis-synthesis filter bank cascade (polyphase representation).

of optimal synthesis filter banks achieving PR is an active research topic. Different algorithms arise depending on the class of inverses [e.g., causal, anticausal or noncausal, finite-impulse response (FIR) or infinite impulse response (IIR)] and the optimality criterion used (e.g., minimal $\mathcal{H}_{2}$ or $\mathcal{H}_{\infty}$ norm). In this paper, we consider causal IIR inverses with minimal weighted $\mathcal{H}_{2}$ norm.

Problem 1 (Weighted Inversion): Suppose we are given $H \in$ $\mathcal{R H}_{\infty}^{m \times l}$ and $W \in \mathcal{R H}_{\infty}^{m \times l_{W}}$ such that $\operatorname{rank}[H(z)]=l<m$ for $1 \leq|z| \leq \infty, \operatorname{rank}\left[\left(I-H(z) H^{\dagger}(z)\right) W(z)\right]$ is constant for $|z|=1$, and $L>0 .{ }^{1}$ Then, we want to find $G_{\text {opt }} \in \mathcal{R} \mathcal{H}_{\infty}^{l \times m}$ such that

$G_{\mathrm{opt}}(z) H(z)=z^{-L} I \quad$ and $\quad\left\|G_{\mathrm{opt}} W\right\|_{2}=\inf _{\substack{\tilde{\sigma} H=L^{-L_{I}} \\ \tilde{G} \in \mathcal{R} \mathcal{H}_{\infty}}}\|\tilde{G} W\|_{2} \cdot$

Let us review some relevant literature. The delay-free version of Problem 1 with constant weight $\left(L=0, W(z) \equiv W_{0}\right)$ has been solved in [5]. This approach is optimal in terms of delay but at the same time usually suffers from large minimal norms. The other extreme, i.e., the noncausal version of Problem $1(L=\infty)$, has been solved only very recently in [6]. While the norms of inverses are often much smaller in the noncausal approach than for causal inverses, there is the problem that the synthesis filter banks cannot be implemented in real-time because the decision delay is infinite, in general. ${ }^{2}$ Our solution finds the middle ground between these two extremes, i.e., we let the filter bank designer choose a tradeoff between decision delay and norm. Some preliminary results on this case have already been given by the authors in [8]. However, the assumptions on the weight in [8] are more restrictive, and some proofs were left open. To the best of our knowledge, the case considered here is

\footnotetext{
${ }^{1}$ Our approach also applies to the case $L=0$ but this requires some straightforward modifications which are not treated in this paper. However, note that the toolbox referenced in Section V implements this case.

${ }^{2}$ To be precise, it is also possible to implement the filter bank as a causal but in general unstable system (see, e.g., [7, Ex. 3.7]. However, these are suitable only for very short input sequences.
} 
still open. Other inversion problems treated in the literature are, e.g., causal IIR inverses with minimal unweighted $\mathcal{H}_{2}$ norm [9], [10] or minimal $\mathcal{H}_{\infty}$ norm [11], [12]. Causal FIR inverses with minimal $\mathcal{H}_{2}$ norm are treated in [13], [14]. The para-pseudoinverse, which in general is noncausal and IIR, is considered in [15]-[17]. Anticausal inversion is discussed in [18] and [19].

Similar to [5], [10], and [11], we will choose a state-space approach to solve the weighted inversion problem. Our algorithm exhibits two interesting features. First, our setting is quite general, so that there may be multiple optimal solutions. The algorithm computes the complete set of solutions. This is an improvement over the results in [5], [6], and [8], where the assumptions enforced uniqueness of optimal inverses, and provides more degrees of freedom to the filter bank designer. Second, the complexity grows only linearly with the decision delay. Thus, also large decision delays become feasible.

The paper is structured as follows. In Section II, we parametrize the stable inverses of $H(z)$, and in Section III we derive the set of optimal stable inverses. The efficiency of the numerical procedures outlined in Section III is improved in Section IV. Numerical examples are given in Section V. The paper ends with a conclusion (Section VI) and an Appendix.

We close this section with a couple of remarks.

Remark 1 (Necessity of Conditions): Not all the assumptions in Problem 1 are necessary; only $\operatorname{rank}[H(z)]=l$ for $1 \leq|z|<\infty$ is. On the other hand, this condition alone is not sufficient. Note that we can still solve Problem 1 approximately if the conditions are not met. If $\left(I-H H^{\dagger}\right) W$ has zeros on $|z|=1$, we can compute an optimal inverse $\tilde{G}$ for $H$ with weight $\tilde{W}:=[W, \epsilon I]$, where $\epsilon>0$ is small. $\tilde{G}$ then minimizes $\|G W\|_{2}^{2}+\epsilon^{2}\|G\|_{2}^{2}$. If $H$ violates the rank condition, we can use the approach given in the next remark (with weight $\epsilon W$ ).

Remark 2 (MMSE Criterion): There are situations where the optimal exact inverse is not desired but an approximate inverse which minimizes the minimum mean-square error (MMSE). Interestingly, this problem can be seen as a special case of Problem 1 [8]. (The opposite is not true.) Suppose that we want to estimate $u$ from measurements $y=H u+W n$ where $u$ and $n$ are spatially and temporally white Gaussian random signals. Introduce the auxiliary systems

$$
\tilde{H}:=\left[\begin{array}{c}
H \\
I
\end{array}\right] \quad \text { and } \quad \tilde{W}:=\left[\begin{array}{cc}
W & 0 \\
0 & I
\end{array}\right]
$$

and compute the corresponding solution $\tilde{G}=[G, R]$ to Problem 1. Then, $\hat{u}=G y$ is a MMSE estimator for $u .^{3}$

Remark 3 (Inverses With Unstable Poles): In the literature, many algorithms compute inverses with unstable poles, e.g., the para-pseudoinverse [15]-[17]. In the time-domain, these inverses can either be implemented as stable systems with twosided impulse response or as unstable systems with one-sided impulse response [7]. ${ }^{4}$ Both variants have their disadvantages. The former cannot be implemented in real-time because the impulse response is in general bi-infinite, which corresponds to an infinite decision delay. The usual remedy is to truncate the

\footnotetext{
${ }^{3}$ The key facts to note are that the MSE is given by $\left\|z^{-L} I-G H\right\|_{2}^{2}+\|G W\|_{2}^{2}$ and that $\tilde{G} \tilde{H}=z^{-L} I$ iff $R=z^{-L} I-G H[8]$.

${ }^{4}$ The latter may require a finite decision delay.
}

impulse response, which introduces errors. On the other hand, the latter variant can be implemented in real-time, but the result usually is unusable because even the slightest error (e.g., finite precision effects) can be amplified ad infinitum.

Remark 4 (Anticausal Inverses): Some papers also consider anticausal inverses [18], [19]. The impulse response of an anticausal inverse is always one-sided. This is similar to causal inverses. The difference is that the impulse response extends backwards in time instead of forwards. Counterintuitively, also anticausal inverses can be implemented in real-time when a certain block processing method is used [18], [19]. (But note that this method cannot be applied to inverses with bi-infinite impulse response like the stable implementation of the para-pseudoinverse.) However, this method needs side information usually not known at the inverse, i.e., the first systems internal state sequence decimated by the block length.

Remark 5 (Spectral Factorization Approach): In this paper, we solve Problem 1 directly. An indirect approach was presented in our previous work [8]. There, spectral factorization was used to reduce the weighted system inversion problem to an unweighted system inversion problem (cf. [8, Prop. 2]). We want to point out that our new approach offers some advantages. The spectral factorization approach is only guaranteed to work if the weight has full row rank on $|z|=1 . .^{5}$ This condition is more restrictive than the one in Problem 1. Another obvious advantage is that the additional spectral factorization is not necessary, which decreases the computational burden.

\section{PARAMEtrization OF the Stable InVERSES}

A major step in the solution of the inversion problem is to convert the constrained optimization problem (1) into an unconstrained optimization problem. Thus, we now derive an explicit parametrization for the set of inverses. The parametrization follows the results in [11] very closely, which is why we only give the main results. Consider a (minimal) state-space realization [1]

$$
H(z)=D+C(z I-A)^{-1} B=\left[\begin{array}{l|l}
A & B \\
\hline C & D
\end{array}\right]
$$

where $A \in \mathbb{C}^{n \times n}$. Since $D=H(\infty)$ has full column rank by assumption, the pseudoinverse $D^{+}:=\left(D^{*} D\right)^{-1} D^{*}$ is well defined. We can find a unitary matrix $D_{\perp} \in \mathbb{C}^{m \times(m-l)}$ such that $D_{\perp} D_{\perp}^{*}=I-D D^{+}$. Then, with $D_{\perp}^{+}:=D_{\perp}^{*}$, the relation

$$
\left[\begin{array}{l}
D^{+} \\
D_{\perp}^{+}
\end{array}\right]\left[\begin{array}{ll}
D & D_{\perp}
\end{array}\right]=\left[\begin{array}{ll}
I & 0 \\
0 & I
\end{array}\right]
$$

holds. Furthermore, a $n \times(m-l)$ matrix $B_{\perp}$ exists such that the matrix $A_{0}:=A-B D^{+} C-B_{\perp} D_{\perp}^{+} C$ is stable, i.e., all eigenvalues are contained inside the complex unit disc. (A suitable $B_{\perp}$ can be computed using pole placement algorithms.) Now, we can extend $H$ into an invertible rational matrix

$$
\left[\begin{array}{ll}
H & H_{\perp}
\end{array}\right]:=\left[\begin{array}{c|cc}
A & B & B_{\perp} \\
\hline C & D & D_{\perp}
\end{array}\right] \in \mathcal{R} \mathcal{H}_{\infty}^{m \times m} .
$$

${ }^{5}$ If the row rank $r$ is lower than $m$, rank reduced spectral factorizations with $m \times r$ spectral factors may be used [20]. However, the resulting unweighted system inversion problem no longer has to be solvable. In particular, if $r<l$, it is always unsolvable because the resulting system is wide. 
This extended matrix has the inverse

$$
\left[\begin{array}{c}
G \\
G_{\perp}
\end{array}\right]:=\left[\begin{array}{c}
D^{+} \\
D_{\perp}^{+}
\end{array}\right]\left[\begin{array}{c|c}
A_{0} & B_{0} \\
\hline C & I
\end{array}\right] \in \mathcal{R H}_{\infty}^{m \times m}
$$

where $B_{0}:=-B D^{+}-B_{\perp} D_{\perp}^{+}$. Analogously to Proposition 4.5 in [11], we now obtain the following parametrization.

Proposition 1: A rational matrix $\tilde{G} \in \mathcal{R} \mathcal{H}_{\infty}^{l \times m}$ satisfies $\tilde{G} H=z^{-L} I$ iff $\tilde{G}=z^{-L} G+K G_{\perp}$ for some $K \in \mathcal{R} \mathcal{H}_{\infty}^{l \times(m-l)}$.

Next, we reformulate this parametrization in the optimal control framework (see, e.g., [1]). This will allow us to use results from optimal control in order to solve our system inversion problem.

Theorem 1 (Parametrization of Inverses): Define a $(n+L l) \times$ $(n+L l)$ matrix $\hat{A}$ and a $(n+L l) \times m$ matrix $\hat{B}$ via

$$
\hat{A}:=\left[\begin{array}{ccccc}
A_{0} & 0_{n \times l} & & & \\
D^{+} C & 0_{l} & & & \\
& I_{l} & 0_{l} & & \\
& & \ddots & \ddots & \\
& & & I_{l} & 0_{l}
\end{array}\right], \hat{B}:=\left[\begin{array}{c}
B_{0} \\
D^{+} \\
0_{l \times m} \\
\vdots \\
0_{l \times m}
\end{array}\right]
$$

as well as a $m \times(n+L l)$ matrix $\hat{C}$ and a $m \times m$ matrix $\hat{D}$ via

$$
\hat{C}:=\left[\begin{array}{lllll}
D_{\perp} D_{\perp}^{+} C & 0_{m \times l} & \ldots & 0_{m \times l} & D
\end{array}\right], \hat{D}:=D_{\perp} D_{\perp}^{+} .
$$

Furthermore, introduce the state-space system

$$
\Sigma_{S}:\left[\begin{array}{c}
\hat{x}_{k+1} \\
z_{k} \\
y_{k}
\end{array}\right]=\left[\begin{array}{ccc}
\hat{A} & \hat{B} & 0 \\
D^{+} \hat{C} & 0 & I_{l} \\
D_{\perp}^{+} \hat{C} & D_{\perp}^{+} & 0
\end{array}\right]\left[\begin{array}{c}
\hat{x}_{k} \\
w_{k} \\
u_{k}
\end{array}\right] .
$$

Then, $\tilde{G} \in \mathcal{R} \mathcal{H}_{\infty}^{l \times m}$ satisfies $\tilde{G} H=z^{-L} I$ iff it is the closedloop transfer function of $\Sigma_{S}$ for an internally stabilizing controller

$$
\Sigma_{K}:\left[\begin{array}{c}
x_{k+1}^{K} \\
u_{k}
\end{array}\right]=\left[\begin{array}{cc}
A_{K} & B_{K} \\
C_{K} & D_{K}
\end{array}\right]\left[\begin{array}{c}
x_{k}^{K} \\
y_{k}
\end{array}\right]
$$

The proof of this theorem is relatively simple when one notes that the control inputs actually do not affect the states, which makes $\mathcal{R} \mathcal{H}_{\infty}^{l \times(m-l)}$ the set of stabilizing controllers, and (3) is applied to simplify the expressions for $D^{+} \hat{C}, D^{+} \hat{D}, D_{\perp}^{+} \hat{C}$, and $D_{\perp}^{+} \hat{D}$.

\section{INVERSION WITH WEIGHTED $\mathcal{H}_{2}$ NORM CRITERION}

We now derive a solution to the inversion problem. We bring in a (minimal) state-space realization of the weight

$$
\begin{aligned}
W(z) & =D_{W}+C_{W}\left(z I-A_{W}\right)^{-1} B_{W} \\
& =\left[\begin{array}{l|r}
A_{W} & B_{W} \\
\hline C_{W} & D_{W}
\end{array}\right]
\end{aligned}
$$

where $A_{W} \in \mathbb{C}^{n_{W} \times n_{W}}$. In Theorem 1, we have reformulated the parametrization $z^{-L} G+K G_{\perp}$ in the optimal control framework. In order to obtain an optimal inverse, we have to find $K_{\text {opt }} \in \mathcal{R H}_{\infty}^{l \times(m-l)}$ such that $\left\|\left(z^{-L} G+K_{\text {opt }} G_{\perp}\right) W\right\|_{2}$ is minimized. Then, $G_{\mathrm{opt}}=z^{-L} G+K_{\mathrm{opt}} G_{\perp}$ solves the inversion problem. The following theorem reformulates this solution approach in the optimal control framework.
Theorem 2 (Parametrization of Weighted Inverses): Define

$$
\begin{aligned}
& \hat{\mathbf{A}}:=\left[\begin{array}{cc}
A_{W} & 0 \\
\hat{B} C_{W} & \hat{A}
\end{array}\right], \quad \hat{\mathbf{B}}:=\left[\begin{array}{c}
B_{W} \\
\hat{B} D_{W}
\end{array}\right] \\
& \hat{\mathbf{C}}:=\left[\begin{array}{ll}
\hat{D} C_{W} & \hat{C}
\end{array}\right], \quad \hat{\mathbf{D}}:=\hat{D} D_{W} .
\end{aligned}
$$

Then, $\tilde{G}_{W}=\tilde{G} W$ is a weighted inverse, i.e., $\tilde{G} \in \mathcal{R} \mathcal{H}_{\infty}^{l \times m}$ and $\tilde{G} H=z^{-L} I$, iff it is the closed-loop transfer function of

$$
\boldsymbol{\Sigma}_{\mathbf{S}}:\left[\begin{array}{c}
\hat{x}_{k+1} \\
z_{k} \\
y_{k}
\end{array}\right]=\left[\begin{array}{ccc}
\hat{\mathbf{A}} & \hat{\mathbf{B}} & 0 \\
D^{+} \hat{\mathbf{C}} & 0 & I_{l} \\
D_{\perp}^{+} \hat{\mathbf{C}} & D_{\perp}^{+} D_{W} & 0
\end{array}\right]\left[\begin{array}{c}
\hat{x}_{k} \\
w_{k} \\
u_{k}
\end{array}\right]
$$

for some internally stabilizing controller (6). Furthermore, the internally stabilizing controller for $\boldsymbol{\Sigma}_{\mathbf{S}}$ that results in the closedloop transfer function $\tilde{G}_{W}=\tilde{G} W$ also internally stabilizes $\Sigma_{S}$ in Theorem 1. When applied to $\Sigma_{S}$, the transfer function of the closed-loop system equals $\tilde{G}$.

Thus, we can compute an optimal $K_{\text {opt }}$ by solving the optimal control problem in Theorem 2, and then obtain a statespace realization of an optimal inverse by computing the closedloop system which results from application of $K_{\mathrm{opt}}$ to $\Sigma_{S}$ as given in Theorem 1. Regarding the proof of Theorem 2, one should note that $\boldsymbol{\Sigma}_{\mathbf{S}}$ simply is the concatenation of $\Sigma_{S}$ and the weight $W$. The rest basically carries over from Theorem 1 .

Although we have now reformulated the critical step of computing an optimal parameter $K_{\text {opt }}$ as an optimal control problem, several issues remain. A basic difficulty is that depending on the weight, $K_{\text {opt }}$ is not necessarily unique. Standard optimal control algorithms make additional assumptions such as $D_{\perp}^{+} D_{W}$ having full rank or $l \geq m-l$ to ensure uniqueness of optimal solutions [1], [21]. However, with the weight in Problem 1, those assumptions may not hold, and thus standard algorithms cannot always be applied. We solve this problem by using the singular control results in [21]. The following algorithm is obtained. (See Appendix A for a derivation.)

\section{Algorithm 1: Naive Inversion Algorithm}

Input: $H, W$ as in Problem 1, with realizations (2), (7), and $L>0$.

Outp.: $G_{\mathrm{opt}} \in \mathcal{R} \mathcal{H}_{\infty}^{l \times m}$ s. t. $G_{\mathrm{opt}} H=z^{-L} I,\left\|G_{\mathrm{opt}} W\right\|_{2}=$ min.

Step 1: Compute a stabilizing solution $6\left(\mathbf{Y}_{2}, \mathbf{L}\right)$ to the discrete time algebraic Riccati system (DTARS)

$$
\left\{\begin{array}{l}
\hat{\mathbf{A}} \mathbf{Y}_{2} \hat{\mathbf{A}}^{*}-\mathbf{Y}_{\mathbf{2}}-\mathbf{L}^{*} \mathbf{P L}+\hat{\mathbf{B}} \hat{\mathbf{B}}^{*}=0 \\
D_{\perp}^{+} D_{W} \hat{\mathbf{B}}^{*}+D_{\perp}^{+} \hat{\mathbf{C}} \mathbf{Y}_{\mathbf{2}} \hat{\mathbf{A}}^{*}+\mathbf{P L}=0 .
\end{array}\right.
$$

$$
\text { Here, } \mathbf{P}=D_{\perp}^{+}\left(D_{W} D_{W}^{*}+\hat{\mathbf{C}} \mathbf{Y}_{2} \hat{\mathbf{C}}^{*}\right) D_{\perp} \text {. }
$$

Step 2: Set $\mathbf{L}_{\mathbf{0}}:=-\mathbf{P}^{+} D_{\perp}^{+} \hat{\mathbf{C}} \mathbf{Y}_{2} \hat{\mathbf{C}}^{*}\left(D^{+}\right)^{*}$, and return

$$
\left[\begin{array}{cc|c}
\hat{A} & 0 & \hat{B} \\
\mathbf{L}^{*} D_{\perp}^{+} \hat{C} & \hat{\mathbf{A}}+\mathbf{L}^{*} D_{\perp}^{+} \hat{\mathbf{C}} & \mathbf{L}^{*} D_{\perp}^{+} \\
\hline\left(D^{+}+\mathbf{L}_{\mathbf{0}}^{*} D_{\perp}^{+}\right) \hat{C} & \left(D^{+}+\mathbf{L}_{\mathbf{0}}^{*} D_{\perp}^{+}\right) \hat{\mathbf{C}} & \mathbf{L}_{\mathbf{0}}^{*} D_{\perp}^{+}
\end{array}\right] \text {. }
$$

The following proposition shows that Problem 1 is always solvable.

Proposition 2: Let the assumptions in Problem 1 hold. Then, a stabilizing solution to the DTARS (8) exists.

$6_{\text {i.e., }} \mathbf{Y}_{\mathbf{2}}=\mathbf{Y}_{\mathbf{2}}^{*}$ and $\hat{\mathbf{A}}^{*}+\hat{\mathbf{C}}^{*} D_{\perp} \mathbf{L}$ stable 
The proof of Proposition 2 is given in Appendix B. If the optimal inverse is unique, ${ }^{7}$ the solution to the DTARS is unique as well. It can then be computed using [22, Th. 5.5.1]. In the nonunique case, the more complicated approach in [22, Th. 6.4.4] can be utilized. Once the DTARS is solved, Theorem 4 in [21] implies the following parametrization for the optimal solutions.

Theorem 3 (Parametrization of Optimal Inverses): Suppose that $\mathbf{P}_{0}^{1 / 2}$ is a basis for the co-image of $\mathbf{P}^{1 / 2}$ (i.e., $\mathbf{P}_{0}^{1 / 2}$ has full and maximal rank and $\mathbf{P}_{0}^{1 / 2} \mathbf{P}^{1 / 2}=0$ ), and set

$$
\left[\begin{array}{c}
C_{11}(z) \\
C_{21}(z)
\end{array}\right]:=\left[\begin{array}{c|c}
\hat{\mathbf{A}}+\mathbf{L}^{*} D_{\perp}^{+} \hat{\mathbf{C}} & \mathbf{L}^{*} \\
\hline D^{+} \hat{\mathbf{C}} & 0 \\
D_{\perp}^{+} \hat{\mathbf{C}} & I
\end{array}\right] .
$$

Then, $G_{\text {opt }} \in \mathcal{R} \mathcal{H}_{\infty}^{l \times m}$ satisfies (1) iff, for some $K \in \mathcal{R} \mathcal{H}_{\infty}$,

$$
G_{\mathrm{opt}}=z^{-L} G+\left(C_{11}+\left(K \mathbf{P}_{0}^{1 / 2}+\mathbf{L}_{\mathbf{0}}^{*}\right) C_{21}\right) G_{\perp} \text {. }
$$

This parametrization may e.g., be used to find an optimal inverse with additionally minimal $\mathcal{H}_{\infty}$ norm.

\section{EFFICIENT INVERSION ALGORITHM}

A serious disadvantage of the naive algorithm is that its costs grow rapidly with the delay $L$ because the dimensions of the coefficient matrices in the DTARS (8) grow linearly with $L$. Thus, computational complexity as well as storage requirements for solving this DTARS grow cubically. In this section, we derive a more sophisticated algorithm, where complexity (and storage requirements) grow only linearly with $L$. The key observation will be that only the first $n+n_{W}$ rows of $\mathbf{Y}_{\mathbf{2}}$ are actually used in the naive algorithm, and that this part of $\mathbf{Y}_{2}$ can be obtained without solving the large DTARS (8). Instead, only a small DTARS has to be solved.

For the rest of the section, let us fix a stabilizing solution $\left(\mathbf{Y}_{\mathbf{2}}, \tilde{\mathbf{L}}\right)$ to the DTARS (8). We partition $\mathbf{Y}_{\mathbf{2}}$ as follows,

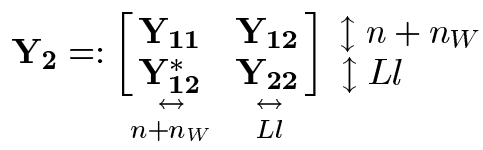

Furthermore, let us introduce

$$
\begin{aligned}
\mathbf{A}_{\mathbf{0}} & :=\left[\begin{array}{cc}
A_{W} & 0 \\
B_{0} C_{W} & A_{0}
\end{array}\right], \mathbf{B}_{\mathbf{0}}:=\left[\begin{array}{c}
B_{W} \\
B_{0} D_{W}
\end{array}\right] \\
\mathbf{C}_{\mathbf{0}}: & =\left[\begin{array}{ll}
C_{W} & C
\end{array}\right] .
\end{aligned}
$$

The next proposition shows how $\mathbf{Y}_{\mathbf{1 1}}$ and $\mathbf{Y}_{\mathbf{1 2}}$ can be obtained from $\mathbf{A}_{\mathbf{0}}, \mathbf{B}_{\mathbf{0}}$, and $\mathbf{C}_{\mathbf{0}}$ without solving the large DTARS (8).

Proposition 3: A stabilizing solution ${ }^{8}(X, F)$ for

$$
\left\{\begin{array}{l}
\mathbf{A}_{\mathbf{0}} X \mathbf{A}_{\mathbf{0}}^{*}-X-F^{*} P F+\mathbf{B}_{\mathbf{0}} \mathbf{B}_{\mathbf{0}}^{*}=0 \\
D_{\perp}^{+} D_{W} \mathbf{B}_{\mathbf{0}}^{*}+D_{\perp}^{+} \mathbf{C}_{\mathbf{0}} X \mathbf{A}_{\mathbf{0}}^{*}+P F=0
\end{array}\right.
$$

\footnotetext{
${ }^{7}$ One can show: the optimal inverse is unique iff $\left(I-H H^{\dagger}\right) W$ has normal rank $m-l$, i.e., $\exists z_{0} \in \mathbb{C}$ s. t. $\operatorname{rank}\left[\left(I-H\left(z_{0}\right) H^{\dagger}\left(z_{0}\right)\right) W\left(z_{0}\right)\right]=m-l$.

8i.e., $X=X^{*}$ and $\mathbf{A}_{\mathbf{0}}^{*}+\mathbf{C}_{\mathbf{0}}^{*} D_{\perp} F$ stable
}

exists under the assumptions in Problem 1. Here, $P=$ $D_{\perp}^{+}\left(D_{W} D_{W}^{*}+\mathbf{C}_{\mathbf{0}} X \mathbf{C}_{\mathbf{0}}^{*}\right) D_{\perp}$. Furthermore, any stabilizing solution satisfies $X=\mathbf{Y}_{\mathbf{1 1}}$, and $\mathbf{Y}_{\mathbf{1 2}}=\left[\mathbf{y}_{\mathbf{1 2}}^{(\mathbf{1})}, \ldots, \mathbf{y}_{\mathbf{1 2}}^{(\mathbf{L})}\right]$ is given by

$$
\begin{aligned}
\mathbf{y}_{\mathbf{1 2}}^{(\mathbf{1})}= & \left(\mathbf{A}_{\mathbf{0}} X \mathbf{C}_{\mathbf{0}}^{*}+\mathbf{B}_{\mathbf{0}} D_{W}^{*}+F^{*} D_{\perp}^{+}\left(D_{W} D_{W}^{*}\right.\right. \\
& \left.\left.+\mathbf{C}_{\mathbf{0}} X \mathbf{C}_{\mathbf{0}}^{*}\right)\right)\left(D^{+}\right)^{*}, \\
\mathbf{y}_{\mathbf{1 2}}^{(\mathbf{k})}= & \left(\mathbf{A}_{\mathbf{0}}+F^{*} D_{\perp}^{+} \mathbf{C}_{\mathbf{0}}\right) \mathbf{y}_{\mathbf{1 2}}^{(\mathbf{k}-\mathbf{1})} \quad(k=2, \ldots, L) .
\end{aligned}
$$

Next, we show that knowing $\mathbf{Y}_{\mathbf{1 1}}$ and $\mathbf{Y}_{\mathbf{1 2}}$ (without knowing $\mathbf{Y}_{\mathbf{2 2}}$ ) is already sufficient to compute suitable $\mathbf{L}$ and $\mathbf{L}_{\mathbf{0}}$.

Proposition 4: Let $\left(\mathbf{Y}_{\mathbf{1 1}}, \mathbf{L}_{\mathbf{1}}\right)$ be a stabilizing solution to the DTARS (12). Also, set $\mathbf{L}:=\left[\mathbf{L}_{\mathbf{1}}, \mathbf{L}_{\mathbf{2}}\right]$, where $\mathbf{L}_{\mathbf{2}}$ is given by the equation shown at the bottom of the page. Then, $\left(\mathbf{Y}_{\mathbf{2}}, \mathbf{L}\right)$ is a stabilizing solution to the DTARS (8). The according $\mathbf{L}_{\mathbf{0}}$ in Algorithm 1 satisfies $\mathbf{L}_{\mathbf{0}}=-P^{+} D_{\perp}^{+} \mathbf{C}_{\mathbf{0}} \mathbf{y}_{\mathbf{1 2}}^{(\mathbf{L})}$.

Both propositions are proven in Appendix C. When we incorporate these results into Algorithm 1, a more efficient algorithm is obtained.

\section{Algorithm 2: Efficient Inversion Algorithm}

Input: $H, W$ as in Problem 1, with realizations (2), (7), and $L>0$.

Outp.: $G_{\text {opt }} \in \mathcal{R} \mathcal{H}_{\infty}^{l \times m}$ s. t. $G_{\mathrm{opt}} H=z^{-L} I,\left\|G_{\mathrm{opt}} W\right\|_{2}=$ min.

Step 1: Compute $\mathbf{Y}_{\mathbf{1 1}}$ and $\mathbf{Y}_{\mathbf{1 2}}$ via Proposition 3.

Step 2: Compute $\mathbf{L}$ and $\mathbf{L}_{\mathbf{0}}$ using Proposition 4.

Step 3: Partition $\hat{A}=:\left[\hat{A}_{1}, \hat{A}_{2}, 0\right]$ and $\hat{\mathbf{A}}=:\left[\hat{\mathbf{A}}_{1}, \hat{\mathbf{A}}_{2}, 0\right]$, and return

$\left[\begin{array}{cccccc|c}\hat{A}_{1} & \hat{A}_{2} & 0 & 0 & 0 & 0 & \hat{B} \\ \mathbf{L}^{*} D_{\perp}^{+} C & 0 & 0 & \hat{\mathbf{A}}_{1}+\mathbf{L}^{*} D_{\perp}^{+} \mathbf{C}_{\mathbf{0}} & \hat{\mathbf{A}}_{2} & 0 & \mathbf{L}^{*} D_{\perp}^{+} \\ \hline \mathbf{L}_{\mathbf{0}}^{*} D_{\perp}^{+} C & 0 & I_{l} & \mathbf{L}_{\mathbf{0}}^{*} D_{\perp}^{+} \mathbf{C}_{\mathbf{0}} & 0 & I_{l} & \mathbf{L}_{\mathbf{0}}^{*} D_{\perp}^{+}\end{array}\right]$.

To see that the storage requirements indeed only grow linearly with the delay $L$, note that $\mathbf{L}^{*} D_{\perp}^{+}$is a $\left(n+n_{W}+L l\right) \times m$ matrix. The matrices $\hat{A}_{2}$ and $\hat{\mathbf{A}}_{2}$ simply are identity matrices augmented by zeros, which do not have to be stored.

\section{NumericAl EXAMPLES}

In this section, we illustrate the use of our results with some numerical examples. Following the idea of reproducible research, all software necessary to recreate our results is provided [23]. In particular, we have implemented the inversion algorithm in a Scilab ${ }^{9}$ toolbox available at http://lsitbx.origo.ethz.ch. The Scilab scripts that where used to create the numerical examples are additional multimedia content to the paper, which can be downloaded using IEEE Xplore (http://ieeexplore.ieee.org). The aforementioned toolbox is required to run them.

\footnotetext{
${ }^{9} \mathrm{Scilab}$ is an open source platform for numerical computation, see http:// www.scilab.org for more information
}

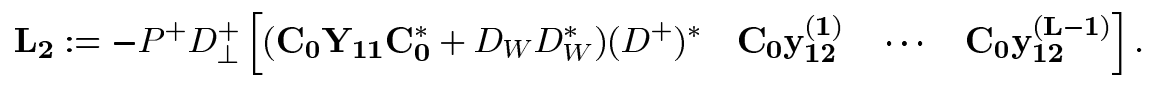




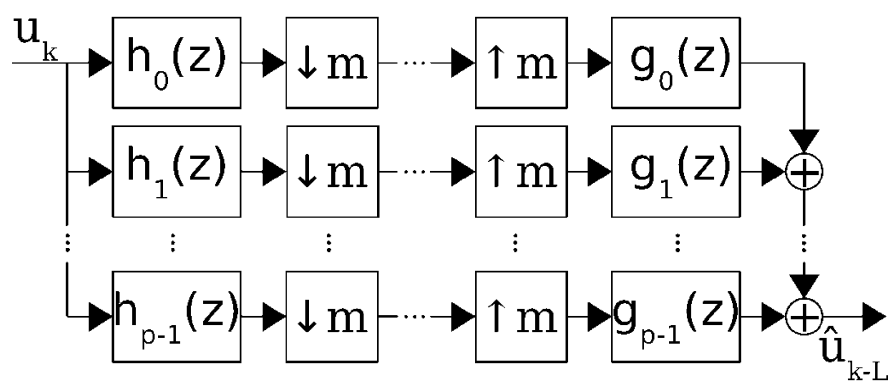

Fig. 2. Perfect reconstruction analysis-synthesis filter bank cascade.

PR Filter Bank Cascade: We revisit Example 1 in [16], where PR filter bank cascades as depicted in Fig. 2 are considered. The sampling factor is $m=2$. We employ $p=3$ filters. The analysis filters are

$$
\begin{aligned}
h_{0}(z) & :=\frac{0.4208 z+0.4208}{z-0.1584} \\
h_{1}(z) & :=\frac{0.2452 z^{2}-0.2452}{z^{2}+0.5095} \\
h_{2}(z) & :=h_{0}(-z) .
\end{aligned}
$$

Let us compute the polyphase matrix of the analysis filter bank, i.e., $H(z)$ in Fig. 1. By [16, eq. (31)]

$$
H(z) \approx\left[\begin{array}{cc}
\frac{0.0666547+0.4208 z}{-0.0250906+z} & \frac{0.4874547 z}{-0.0250906+z} \\
\frac{-0.2452+0.2452 z}{0.5095+z} & 0 \\
\frac{0.0666547+0.4208 z}{-0.0250906+z} & \frac{-0.4874547 z}{-0.0250906+z}
\end{array}\right] .
$$

Now, let $G(z)=\left[g_{i, j}(z)\right]_{i, j}$ denote an optimal left-inverse for $H(z)$ (note that $G$ depends on the delay $L$ ). The synthesis filters in Fig. 2 then are given by $g_{k}(z):=\sum_{j=0}^{m-1} z^{-j} g_{k, j}\left(z^{m}\right)[16$, eq. (9)].

The norms of the optimal inverses $G(z)$ are approximately 2.1324, 1.9896, 1.9774, and 1.9771 for $L=0, L=1, L=2$, and $L=\infty$, respectively. (Here, $L=0$ was solved with a modified version of Algorithm 1. The cases $L=1,2$ were solved with Algorithm 2, while for $L=\infty$ we used the nonrealizable para-pseudoinverse from [6].) Thus, the performance of the nonrealizable para-pseudoinverse can be achieved by causal inverses even for small delays. In order to visualize the filter banks, we have plotted normalized versions of the average polyphase magnitudes of analysis and synthesis filter banks in Fig. 5, i.e., $|\bar{h}(f)|:=1 / p \sum_{k=0}^{p-1}\left|h_{k}\left(e^{2 \pi i f}\right)\right|$ and $|\bar{g}(f)|:=1 / p \sum_{k=0}^{p-1}\left|g_{k}\left(e^{2 \pi i f}\right)\right|$. Note how closely the average polyphase magnitude of the optimal inverse with delay $L=2$ already matches the average polyphase magnitude of the nonrealizable para-pseudoinverse $(L=\infty)$.

Multirate Transceiver: We revisit Example 1 in [5] (originally due to [24, Ex. 2]), where multirate transceivers as depicted in Fig. 3 are considered. The $\left\{f_{k}\right\}$ are precoding filters, $h$ is a communications channel, and the $\left\{g_{k}\right\}$ are the equalization filters. The sampling factors are $p=4$ and $m=3$. The channel

$$
\begin{aligned}
h(z):=1-0.3 z^{-1}+ & 0.5 z^{-2}-0.4 z^{-3} \\
& +0.1 z^{-4}-0.02 z^{-5}+0.3 z^{-6}-0.1 z^{-7}
\end{aligned}
$$

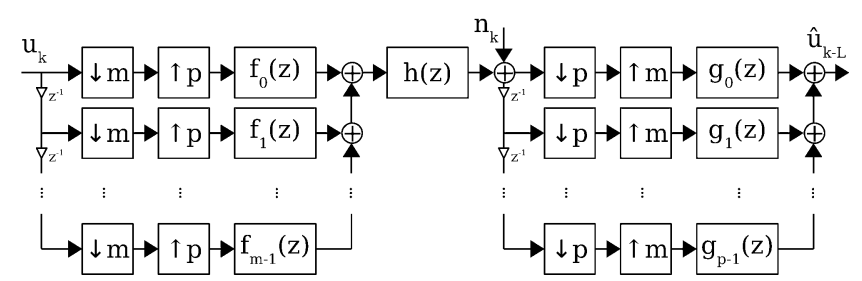

Fig. 3. Multirate transceiver.

has four polyphase components

$$
\begin{aligned}
& h_{0}(z):=1+0.1 z^{-1}, h_{1}(z):=-0.3-0.02 z^{-1} \\
& h_{2}(z):=0.5+0.3 z^{-1}, h_{3}(z):=-0.4-0.1 z^{-1} .
\end{aligned}
$$

The precoding filters are given by $f_{k}(z)$ := $\sum_{k=0}^{m-1} z^{-j} f_{k, j}\left(z^{p}\right)$, where

$$
F(z):=\left[f_{i, j}(z)\right]_{i, j}:=\tilde{F}(z) \Omega^{-1}(z) .
$$

Here, $\tilde{F}(z):=\left[I_{m}, 0\right]^{\sim}$ is an initial precoder, and $\Omega(z)$ is a co-spectral factor of $\tilde{F}^{\sim}(z) \tilde{H}^{\sim}(z) \tilde{H}(z) \tilde{F}(z)$ with $\tilde{H}(z)$ given by $[5$, eq. (9)]

$$
\tilde{H}(z):=\left[\begin{array}{cccc}
h_{0}(z) & z^{-1} h_{3}(z) & z^{-1} h_{2}(z) & z^{-1} h_{1}(z) \\
h_{1}(z) & h_{0}(z) & z^{-1} h_{3}(z) & z^{-1} h_{2}(z) \\
h_{2}(z) & h_{1}(z) & h_{0}(z) & z^{-1} h_{3}(z) \\
h_{3}(z) & h_{2}(z) & h_{1}(z) & h_{0}(z)
\end{array}\right]
$$

i.e., $\Omega, \Omega^{-1} \quad \in \quad \mathcal{R H}_{\infty}$ and $\Omega^{\sim}(z) \Omega(z) \quad=$ $\tilde{F}^{\sim}(z) \tilde{H}^{\sim}(z) \tilde{H}(z) \tilde{F}(z)$. Combination of precoder and channel gives us the polyphase matrix of a virtual analysis filter bank, $H(z):=\tilde{H}(z) F(z)[5$, eq. (17)]. Now, let $G(z)=\left[g_{i, j}(z)\right]_{i, j}$ denote an optimal left inverse for $H(z)$ (note that $G$ depends on $L$ ). The resulting equalization filters for Fig. 3 are given by $g_{k}(z):=\sum_{j=0}^{m-1} z^{-j} g_{k, j}\left(z^{m}\right)$.

In [5, Ex. 1], the equalization filter bank was obtained for the delay-free case $L=0$. The resulting norm was 1.7856 . In the case $L=0$, we obtained a very similar norm of 1.7799 . 10 When we use Algorithm 2 to increase the decision delay to $L=1, L=2$, and $L=5$, the norms drop further to $1.7481,1.7375$, and 1.7324 , respectively. The optimal nonrealizable filter bank given in [6, Th. 1] (which corresponds to $L=$ $\infty$ ) achieves a norm of 1.7321 . Thus, the performance of the optimal nonrealizable filter bank can again be nearly achieved even with quite small decision delays. We have also again plotted normalized versions of the average polyphase magnitudes of channel, precoder and various equalizers in Fig. 5, i.e., $|\bar{h}(f)|$ and $|\bar{g}(f)|$ as defined in the previous example, and $|\bar{f}(f)|:=$ $1 / p \sum_{k=0}^{p-1}\left|f_{k}\left(e^{2 \pi i f}\right)\right|$. Note that our plots of $\bar{f}(f)$ and $\bar{g}(f)$ (for $L=0$ ) differ from [5, Fig. 2] because the spectral factorization used in the computation of the transmitter filter bank is not unique. Fig. 5 illustrates how the finite-delay equalization filter banks converge towards the nonrealizable $(L=\infty)$ filter bank when the delay $L$ is increased. Also note how close the average magnitude of the equalization filter bank $(L=5)$ already is to that of the optimal nonrealizable filter bank.

\footnotetext{
${ }^{10}$ In fact, both errors should be equal. The difference has numerical reasons.
} 


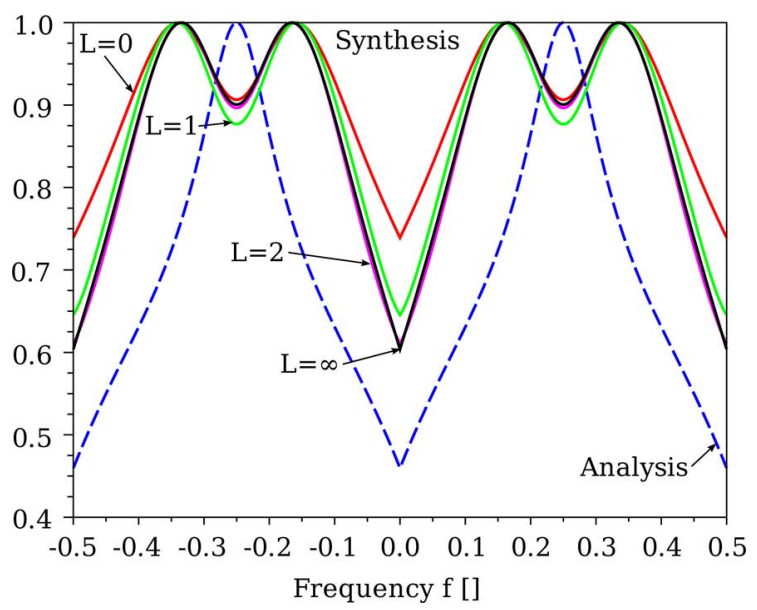

Fig. 4. Normalized average polyphase magnitudes of analysis filter bank (dashed) and synthesis filter banks (solid).

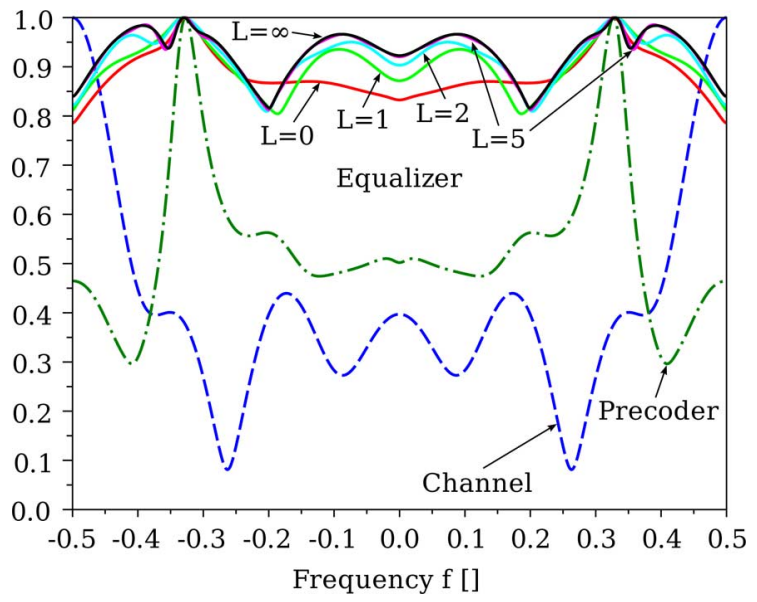

Fig. 5. Normalized average polyphase magnitudes of channel (dashed), precoding filter bank (dash-dotted), and equalization filter banks (solid).

Nonunique Optimal Inverses: We consider Problem 1 with $L=1$,

$H(z):=\left[\begin{array}{l|l}A & B \\ \hline C & D\end{array}\right]:=\left[\begin{array}{l|l}0.5 & 1 \\ \hline-0.25 & -0.5 \\ -0.5 & -1 \\ -0.75 & -1.5\end{array}\right]=\frac{z}{1-2 z}\left[\begin{array}{l}1 \\ 2 \\ 3\end{array}\right]$ $W(z):=\left[\begin{array}{l|l}A_{W} & B_{W} \\ \hline C_{W} & D_{W}\end{array}\right]:=\left[\begin{array}{c|c}0 & 1 \\ \hline 1 & 0 \\ 0 & 0.1 \\ 0 & 0.1\end{array}\right]=\left[\begin{array}{c}z^{-1} \\ 0.1 \\ 0.1\end{array}\right]$.

Following Section II, we first compute an extension matrix (cf. (3))

$$
D_{\perp} \approx\left[\begin{array}{cc}
-0.9636241 & 0 \\
0.1482499 & -0.8320503 \\
0.2223748 & 0.5547002
\end{array}\right]
$$

with $D_{\perp}^{+}=D_{\perp}^{*}$ from the singular value decomposition of $I-$ $D D^{+}$. Since $B=1$ and $C=0.5 D$, we see that $A_{0}=A-$ $B D^{+} C=0$ is stable. Thus, we can choose $B_{\perp}=[0,0]$, which gives $B_{0}=-B D^{+}=-D^{+}$. Thus, in light of (5), we have completed computation of the inverse $G$ and its extension matrix $G_{\perp}$. Next, we compute (11),

$$
\begin{aligned}
\mathbf{A}_{\mathbf{0}} & \approx\left[\begin{array}{cc}
0 & 0 \\
0.1428571 & 0
\end{array}\right], \quad \mathbf{B}_{0} \approx\left[\begin{array}{c}
1 \\
0.0714286
\end{array}\right], \\
\mathbf{C}_{\mathbf{0}} & =\left[\begin{array}{cc}
1 & -0.25 \\
0 & -0.5 \\
0 & -0.75
\end{array}\right] .
\end{aligned}
$$

Theorem 4.1 in [25] (with perturbation $\epsilon=10^{-10}$ ) shows that

$$
\mathbf{Y}_{\mathbf{1 1}} \approx\left[\begin{array}{ll}
0 & 0 \\
0 & 0
\end{array}\right]
$$

and

$$
\mathbf{L}_{\mathbf{1}} \approx\left[\begin{array}{cc}
0 & 0.1482499 \\
36.055513 & 2.773501
\end{array}\right]
$$

solve the DTARS (12). Then, $\mathbf{y}_{\mathbf{1 2}}^{(\mathbf{1})} \approx 0$, and, by Proposition 4 ,

$$
\mathbf{L}=\left[\begin{array}{ll}
\mathbf{L}_{\mathbf{1}} & \mathbf{L}_{\mathbf{2}}
\end{array}\right] \approx\left[\begin{array}{ccc}
0 & 0.1482499 & -0.1482499 \\
36.055513 & 2.773501 & -2.7735011
\end{array}\right] .
$$

Finally, we can solve for the set of optimal inverses. The matrix $\mathbf{P}=P$ and a basis $\mathbf{P}_{\mathbf{0}}^{\mathbf{1} / \mathbf{2}}$ of the co-image are given by

$$
\begin{aligned}
\mathbf{P} & \approx\left[\begin{array}{cc}
0.0013736 & -0.0010279 \\
-0.0010279 & 0.0007692
\end{array}\right] \\
\mathbf{P}_{\mathbf{0}}^{\mathbf{1 / 2}} & \approx\left[\begin{array}{ll}
0.5991447 & 0.8006408
\end{array}\right] .
\end{aligned}
$$

$$
\begin{aligned}
& C(z) \\
& \approx\left[\begin{array}{ccc|cc}
0 & 0 & 0 & 0 & 36.055513 \\
0 & 0 & 0 & 0.1482499 & 2.773501 \\
0 & 0.5 & 0 & -0.1482499 & -2.7735011 \\
\hline 0 & 0 & 1 & 0 & 0 \\
-0.9636241 & 0 & 0 & 1 & 0 \\
0 & 0 & 0 & 0 & 1
\end{array}\right] \\
& \approx\left[\begin{array}{ccc}
\frac{0.0741249-0.1482499 z}{z^{2}} & \frac{1.3867535-2.7735011 z}{-0.0000002 z+z^{2}} \\
1 & \frac{-34.743961}{z} \\
0 & & 1
\end{array}\right] .
\end{aligned}
$$

After some further computations, $C:=\left[C_{11}^{\sim}, C_{21}^{\sim}\right]^{\sim}$ is obtained [see the equation shown at the bottom of the next page]. The set of optimal inverses is now given by (9). The canonical choice $K(z)=0$ gives the optimal inverse $z^{-1} G+\left(C_{11}+\mathbf{L}_{0}^{*} C_{21}\right) G_{\perp}$.

\section{CONCLUSION}

In this paper, optimal stable inversion of rational matrices with weighted $\mathcal{H}_{2}$ norm criterion was considered. In contrast to known algorithms, our algorithm allows to tradeoff between decision delay and norm of the inverse. Linear complexity in the delay allows implementation also of large delays. Furthermore, the algorithm handles many singular problems with nonunique optimal inverses. In such cases, the set of optimal inverses is parametrized. Numerical examples illustrated applications in filter bank theory. 


\section{APPENDIX A \\ DERIVATION OF ALGORITHM 1}

In order to solve the optimal control problem in Theorem 2, we use the results from [21]. According to [21], two full information control (FIC) problems have to be solved. It is simple to check that the triple $\left(X, F, F_{0}\right)=\left(0,-D^{+} \hat{\mathbf{C}}, 0\right)$ (notation as in [21]) is the optimal solution to the first FIC problem

$$
\left[\begin{array}{c|cc}
\hat{\mathbf{A}} & \hat{\mathbf{B}} & 0 \\
\hline D^{+} \hat{\mathbf{C}} & 0 & I
\end{array}\right] \text {. }
$$

Thus, the second FIC problem is

$$
\left[\begin{array}{c|cc}
\hat{\mathbf{A}}^{*} & \left(D^{+} \hat{\mathbf{C}}\right)^{*} & \left(D_{\perp}^{+} \hat{\mathbf{C}}\right)^{*} \\
\hline \hat{\mathbf{B}}^{*} & 0 & \left(D_{\perp}^{+} D_{W}\right)^{*}
\end{array}\right] .
$$

Polyakov [21] gives a solution $\left(\mathbf{Y}, \mathbf{L}^{*}, \mathbf{L}_{\mathbf{0}}^{*}\right)$ in terms of maximal stabilizing deflating subspaces for the matrix pencil

$\lambda\left[\begin{array}{ccc}I & 0 & 0 \\ 0 & -\hat{\mathbf{A}} & 0 \\ 0 & -D_{\perp}^{+} \hat{\mathbf{C}} & 0\end{array}\right]-\left[\begin{array}{ccc}\hat{\mathbf{A}}^{*} & 0 & \hat{\mathbf{C}}^{*} D_{\perp} \\ \hat{\mathbf{B}} \hat{\mathbf{B}}^{*} & -I & \hat{\mathbf{B}} D_{W}^{*} D_{\perp} \\ D_{\perp}^{+} D_{W} \hat{\mathbf{B}}^{*} & 0 & D_{\perp}^{+} \hat{\mathbf{C}}^{*} D_{\perp}\end{array}\right]$.

Theorem 6.4.4 in [22] shows that equivalently the DTARS (8) can be considered. Thus, we can apply Theorem 3 in [21], which results in the optimal controller

$$
\left[\begin{array}{c|c}
\hat{\mathbf{A}}+\mathbf{L}^{*} D_{\perp}^{*} \hat{\mathbf{C}} & \mathbf{L}^{*} \\
\hline\left(D^{+}+\mathbf{L}_{0}^{*} D_{\perp}^{+}\right) & \mathbf{L}_{\mathbf{0}}^{*}
\end{array}\right]
$$

Finally, we compute the closed-loop system resulting from applying this controller to $\Sigma_{S}$ as given in Theorem 1 (cf. [22, p. 40]) to obtain Step 2) of the algorithm.

\section{APPENDIX B \\ PROOF OF PROPOSITION 2}

Proof: We have that $\operatorname{rank}\left[\left(I-H(z) H^{\dagger}(z)\right) W(z)\right]$ is constant for all $|z|=1$ by the assumptions in Problem 1 . Now, note that the kernels of $G_{\perp}(z) W(z)$ and $\left(I-H(z) H^{\dagger}(z)\right) W(z)$ coincide on $|z|=1$. To see this, suppose $u_{2}:=G_{\perp}\left(z_{0}\right) W\left(z_{0}\right) x=$ 0 for some $\left|z_{0}\right|=1$ and $x \in \mathbb{C}^{l_{W} \times 1}$. Then, with $u_{1}:=$ $G\left(z_{0}\right) W\left(z_{0}\right) x$, we have

$$
\left[\begin{array}{l}
u_{1} \\
u_{2}
\end{array}\right]=\left[\begin{array}{c}
G\left(z_{0}\right) \\
G_{\perp}\left(z_{0}\right)
\end{array}\right] W\left(z_{0}\right) x .
$$

Since (5) is the inverse of (4), it follows that

$$
W\left(z_{0}\right) x=\left[\begin{array}{ll}
H\left(z_{0}\right) & H_{\perp}\left(z_{0}\right)
\end{array}\right]\left[\begin{array}{l}
u_{1} \\
u_{2}
\end{array}\right] \stackrel{\left(u_{2}=0\right)}{=} H\left(z_{0}\right) u_{1} .
$$

This implies $\left(I-H\left(z_{0}\right) H^{\dagger}\left(z_{0}\right)\right) W\left(z_{0}\right) x=(I-$ $\left.H\left(z_{0}\right) H^{\dagger}\left(z_{0}\right)\right) H\left(z_{0}\right) u_{1}=u_{1}-u_{1}=0$. On the other hand,

$$
\begin{aligned}
0 & =\left(I-H\left(z_{0}\right) H^{\dagger}\left(z_{0}\right)\right) W\left(z_{0}\right) x \\
& =\left(I-H\left(z_{0}\right) H^{\dagger}\left(z_{0}\right)\right)\left(H\left(z_{0}\right) u_{1}+H_{\perp}\left(z_{0}\right) u_{2}\right) \\
& =H_{\perp}\left(z_{0}\right) u_{2}-H\left(z_{0}\right) H^{\dagger}\left(z_{0}\right) H_{\perp}\left(z_{0}\right) u_{2}
\end{aligned}
$$

$$
\begin{aligned}
\Rightarrow & G_{\perp}\left(z_{0}\right) H_{\perp}\left(z_{0}\right) u_{2} \\
& =\underbrace{G_{\perp}\left(z_{0}\right) H\left(z_{0}\right)}_{=0} H^{\dagger}\left(z_{0}\right) H_{\perp}\left(z_{0}\right) u_{2}=0 \\
\Rightarrow & G_{\perp}\left(z_{0}\right) W\left(z_{0}\right) x \\
& =\underbrace{G_{\perp}\left(z_{0}\right) H\left(z_{0}\right)}_{=0} u_{1}+\underbrace{G_{\perp}\left(z_{0}\right) H_{\perp}\left(z_{0}\right) u_{2}}_{=0}=0 .
\end{aligned}
$$

We see that $\operatorname{rank}\left[\left(I-H(z) H^{\dagger}(z)\right) W(z)\right]=$ $\operatorname{rank}\left[G_{\perp}(z) W(z)\right]$ for all $|z|=1$ because $\operatorname{rank}[\Phi]=m-\operatorname{dim}(\operatorname{kernel}[\Phi])$ for any $\Phi \in \mathbb{C}^{m \times m}$. Since

$$
G_{\perp}(z) W(z)=\left[\begin{array}{c|c}
\hat{\mathbf{A}} & \hat{\mathbf{B}} \\
\hline D_{\perp}^{+} \hat{\mathbf{C}} & D_{\perp}^{+} D_{W}
\end{array}\right],
$$

Theorem 3.2 in [25] now implies that the equation $\hat{\mathbf{A}} \mathbf{Y}_{2} \hat{\mathbf{A}}^{*}$ $\mathbf{Y}_{\mathbf{2}}-\left(\hat{\mathbf{B}} D_{W}^{*} D_{\perp}+\hat{\mathbf{A}} \mathbf{Y}_{\mathbf{2}} \hat{\mathbf{C}}^{*} D_{\perp}\right)^{*} \mathbf{P}^{+}\left(D_{\perp}^{+} D_{W} \hat{\mathbf{B}}^{*}+\right.$ $\left.D_{\perp}^{+} \hat{\mathbf{C}} \mathbf{Y}_{2} \hat{\mathbf{A}}^{*}\right)+\hat{\mathbf{B}} \hat{\mathbf{B}}^{*}=0$, where $\mathbf{P}$ is given in Algorithm 1, has a solution $\mathbf{Y}_{\mathbf{2}}=\mathbf{Y}_{\mathbf{2}}^{*}$ such that $\hat{\mathbf{A}}^{*}+\hat{\mathbf{C}}^{*} D_{\perp} \mathbf{L}$ is stable for some $\mathbf{L}$. As noted in [25, p. 840], this solution satisfies kernel $[\mathbf{P}] \subset \operatorname{kernel}\left[\hat{\mathbf{B}} D_{W}^{*} D_{\perp}+\hat{\mathbf{A}} \mathbf{Y}_{2} \hat{\mathbf{C}}^{*} D_{\perp}\right]$. Thus, by [22, p. 201], there exists a stabilizing solution to the DTARS (8).

\section{APPENDIX C}

PROOF OF THE PROPOSITIONS 3 AND 4

Lemma 1: We have

$$
\begin{aligned}
& D_{\perp}^{+} \hat{\mathbf{C}} \mathbf{Y}_{2} \hat{\mathbf{C}}^{*} D_{\perp}=D_{\perp}^{+} \mathbf{C}_{\mathbf{0}} \mathbf{Y}_{11} \mathbf{C}_{\mathbf{0}}^{*} D_{\perp} \\
& D_{\perp}^{+} \hat{\mathbf{C}} \mathbf{Y}_{\mathbf{2}} \hat{\mathbf{C}}^{*}\left(D^{+}\right)^{*}=D_{\perp}^{+} \mathbf{C}_{\mathbf{0}} \mathbf{Y}_{\mathbf{1 2}}\left[\begin{array}{c}
0 \\
I_{l}
\end{array}\right] \\
& D_{\perp}^{+} \hat{\mathbf{C}} \mathbf{Y}_{\mathbf{2}} \hat{\mathbf{A}}^{*}=D_{\perp}^{+} \mathbf{C}_{\mathbf{0}}\left[\begin{array}{c}
\mathbf{A}_{\mathbf{0}} \mathbf{Y}_{\mathbf{1 1}} \\
D^{+} \mathbf{C}_{\mathbf{0}} \mathbf{Y}_{\mathbf{1 1}} \\
{\left[\begin{array}{cc}
I_{(L-1) l} & 0
\end{array}\right] \mathbf{Y}_{\mathbf{1 2}}^{*}}
\end{array}\right]^{*} \text {. }
\end{aligned}
$$

Proof: Apply (3) to the definition of $\hat{\mathbf{C}}$ to see that

$$
D_{\perp}^{+} \hat{\mathbf{C}}=\left[\begin{array}{ll}
D_{\perp}^{+} \mathbf{C}_{\mathbf{0}} & 0
\end{array}\right], D^{+} \hat{\mathbf{C}}=\left[\begin{array}{ll}
0 & I
\end{array}\right] .
$$

Then, the computation is straightforward.

Proof: (of Proposition 3) Remember that with (10) and

$$
\tilde{\mathbf{L}}=:\left[\begin{array}{ll}
\tilde{\mathbf{L}}_{1} & \tilde{\mathbf{L}}_{2}
\end{array}\right]=:\left[\begin{array}{llll}
\tilde{\mathbf{L}}_{1} & \tilde{\mathbf{l}}^{(\mathbf{1})} & \ldots & \tilde{\mathbf{l}}^{(\mathbf{L})}
\end{array}\right]
$$

we have fixed a stabilizing solution $\left(\mathbf{Y}_{\mathbf{2}}, \tilde{\mathbf{L}}\right)$ to (8). We show existence of a stabilizing solution to (12) by proving that $\left(\mathbf{Y}_{\mathbf{1 1}}, \tilde{\mathbf{L}}_{1}\right)$ is such a solution. Using (13), it is straightforward to show that $\mathbf{P}=P$ as soon as $X=\mathbf{Y}_{\mathbf{1 1}}$. To see that $\left(\mathbf{Y}_{\mathbf{1 1}}, \tilde{\mathbf{L}}_{1}\right)$ solves the small DTARS (12), now check that

$$
\begin{aligned}
\hat{\mathbf{A}} \mathbf{Y}_{\mathbf{2}} \hat{\mathbf{A}}^{*} & =\left[\begin{array}{cc}
\mathbf{A}_{\mathbf{0}} \mathbf{Y}_{\mathbf{1 1}} \mathbf{A}_{\mathbf{0}}^{*} & \star \\
\star & \star
\end{array}\right], \mathbf{L}^{*} \mathbf{P L}=\left[\begin{array}{cc}
\tilde{\mathbf{L}}_{\mathbf{1}}^{*} P \tilde{\mathbf{L}}_{\mathbf{1}} & \star \\
\star & \star
\end{array}\right], \\
\hat{\mathbf{B}} \hat{\mathbf{B}}^{*} & =\left[\begin{array}{cc}
\mathbf{B}_{0} \mathbf{B}_{0}^{*} & \star \\
\star & \star
\end{array}\right],
\end{aligned}
$$


and apply these equations and Lemma 1 to (8). Next, let us check that $\mathbf{A}_{\mathbf{0}}^{*}+\mathbf{C}_{\mathbf{0}}^{*} D_{\perp} \tilde{\mathbf{L}}_{\mathbf{1}}$ is stable. Therefore, note that by assumption

$$
\hat{\mathbf{A}}^{*}+\hat{\mathbf{C}}^{*} D_{\perp} \tilde{\mathbf{L}} \stackrel{(13)}{=}\left[\begin{array}{cc}
\mathbf{A}_{\mathbf{0}}^{*} & \star \\
0 & \star
\end{array}\right]+\left[\begin{array}{c}
\mathbf{C}_{\mathbf{0}}^{*} D_{\perp} \\
0
\end{array}\right]\left[\begin{array}{ll}
\tilde{\mathbf{L}}_{\mathbf{1}} & \tilde{\mathbf{L}}_{\mathbf{2}}
\end{array}\right]
$$

is stable. By [26, Lem. 7.1.1], then also $\mathbf{A}_{\mathbf{0}}^{*}+\mathbf{C}_{\mathbf{0}}^{*} D_{\perp} \tilde{\mathbf{L}}_{\mathbf{1}}$ is stable. The fact that $\mathbf{Y}_{\mathbf{1 1}}=\mathbf{Y}_{11}^{*}$ follows trivially from $\mathbf{Y}_{\mathbf{2}}=\mathbf{Y}_{2}^{*}$. The partial uniqueness result [22, Pro. 3.6.3] shows that $X=\mathbf{Y}_{\mathbf{1 1}}$ for every other stabilizing solution $(X, F)$.

Now, note that because of Lemma 1 and because both $\left(\mathbf{Y}_{\mathbf{1 1}}, \tilde{\mathbf{L}}_{\mathbf{1}}\right)$ and $(X, F)$ are stabilizing solutions to (12), $\mathbf{Y}_{\mathbf{1 1}}=X$, and $\mathbf{P}=P$, we have $\mathbf{P} \tilde{\mathbf{L}}_{\mathbf{1}}=$ $-\left(D_{\perp}^{+} D_{W} \mathbf{B}_{0}^{*}+D_{\perp}^{+} \mathbf{C}_{\mathbf{0}} \mathbf{Y}_{\mathbf{1 1}} \mathbf{A}_{\mathbf{0}}^{*}\right)=P F$ and $\tilde{\mathbf{L}}_{1}^{*} \mathbf{P} \tilde{\mathbf{L}}_{1}=$ $-\left(\mathbf{A}_{\mathbf{0}} \mathbf{Y}_{\mathbf{1 1}} \mathbf{A}_{\mathbf{0}}^{*}-\mathbf{Y}_{\mathbf{1 1}}+\mathbf{B}_{\mathbf{0}} \mathbf{B}_{\mathbf{0}}^{*}\right)=F^{*} P F$. Additionally using $\mathbf{P}=\mathbf{P}^{*}$, we obtain

$$
\mathbf{P} \tilde{\mathbf{L}}=\mathbf{P}\left[\begin{array}{ll}
F & \tilde{\mathbf{L}}_{2}
\end{array}\right] \quad \text { and } \quad \tilde{\mathbf{L}}^{*} \mathbf{P} \tilde{\mathbf{L}}=\left[\begin{array}{ll}
F & \tilde{\mathbf{L}}_{\mathbf{2}}
\end{array}\right]^{*} \mathbf{P}\left[\begin{array}{ll}
F & \tilde{\mathbf{L}}_{2}
\end{array}\right]
$$

Next, we derive the recursions for the $\mathbf{y}_{\mathbf{1 2}}^{(\mathbf{k})}$. Let us partition

$$
\hat{\mathbf{A}}=\left[\begin{array}{cc}
\mathbf{A}_{11} & 0 \\
0 & \mathbf{A}_{22}
\end{array}\right], \hat{\mathbf{B}}=\left[\begin{array}{c}
\mathbf{B}_{1} \\
0
\end{array}\right]
$$

where

$$
\begin{aligned}
& \mathbf{A}_{\mathbf{1 1}}:=\left[\begin{array}{c}
\mathbf{A}_{\mathbf{0}} \\
D^{+} \mathbf{C}_{\mathbf{0}}
\end{array}\right], \mathbf{B}_{\mathbf{1}}:=\left[\begin{array}{c}
\mathbf{B}_{\mathbf{0}} \\
D^{+} D_{W}
\end{array}\right], \\
& \mathbf{A}_{\mathbf{2 2}}:=\left[\begin{array}{ll}
I_{(L-1) l} & 0_{(L-1) l \times l}
\end{array}\right] .
\end{aligned}
$$

Then, the first line of (8) for $\left(\mathbf{Y}_{\mathbf{2}}, \tilde{\mathbf{L}}\right)$ together with (16) gives

$$
\begin{aligned}
& \mathbf{Y}_{2}=\hat{\mathbf{A}} \mathbf{Y}_{\mathbf{2}} \hat{\mathbf{A}}^{*}-\tilde{\mathbf{L}}^{*} \mathbf{P} \tilde{\mathbf{L}}+\hat{\mathbf{B}} \hat{\mathbf{B}}^{*} \\
& =\left[\begin{array}{cc}
\mathbf{A}_{11} \mathbf{Y}_{11} \mathbf{A}_{11}^{*} & \mathbf{A}_{11} \mathbf{Y}_{12} \mathbf{A}_{22}^{*} \\
\star & \star
\end{array}\right] \\
& -\left[\begin{array}{cc}
F^{*} \mathbf{P} F & F^{*} \mathbf{P} \tilde{\mathbf{L}}_{\mathbf{2}} \\
\star & \star
\end{array}\right]+\left[\begin{array}{cc}
\mathbf{B}_{\mathbf{1}} \mathbf{B}_{\mathbf{1}}^{*} & 0 \\
0 & 0
\end{array}\right]
\end{aligned}
$$

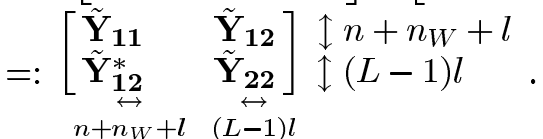

Partition $\tilde{\mathbf{Y}}_{\mathbf{1 2}}=\left[\tilde{\mathbf{y}}_{\mathbf{1 2}}^{(\mathbf{1})}, \ldots, \tilde{\mathbf{y}}_{\mathbf{1 2}}^{(\mathbf{L}-\mathbf{1})}\right]$ into $L-1$ blocks of size $\left(n+n_{W}+l\right) \times l$, and note that $\mathbf{Y}_{\mathbf{1 2}}$ and $\tilde{\mathbf{Y}}_{\mathbf{1 1}}$ overlap because $\mathbf{A}_{\mathbf{1 1}}$ is nonsquare,

$$
\left[\begin{array}{c|c}
\mathbf{Y}_{11} & \mathbf{Y}_{12} \\
\hline \mathbf{Y}_{12}^{*} & \mathbf{Y}_{22}
\end{array}\right]=\left[\begin{array}{c|c}
\tilde{\mathbf{Y}}_{11} & \tilde{\mathbf{Y}}_{12} \\
\hline \tilde{\mathbf{Y}}_{12}^{*} & \tilde{\mathbf{Y}}_{22}
\end{array}\right] .
$$

( $\mathbf{Y}_{\mathbf{1 1}}$ and $\tilde{\mathbf{Y}}_{\mathbf{1 1}}$ have different sizes.) Thus, $\mathbf{y}_{\mathbf{1 2}}^{(\mathbf{1})}$ is the upper right block of $\tilde{\mathbf{Y}}_{\mathbf{1 1}}$. Applying (17) shows us a initial condition for the recursion, i.e.,

$$
\begin{aligned}
\mathbf{y}_{\mathbf{1 2}}^{(\mathbf{1})} & =\left[\begin{array}{ll}
I_{n+n_{W}} & 0
\end{array}\right] \tilde{\mathbf{Y}}_{11}\left[\begin{array}{c}
0 \\
I_{l}
\end{array}\right] \\
& =\mathbf{A}_{\mathbf{0}} \mathbf{Y}_{\mathbf{1 1}} \mathbf{C}_{\mathbf{0}}^{*}\left(D^{+}\right)^{*}-F^{*} \mathbf{P} \tilde{\mathbf{l}}^{(\mathbf{1})}+\mathbf{B}_{\mathbf{0}} D_{W}^{*}\left(D^{+}\right)^{*}
\end{aligned}
$$

Again using (17), we obtain the remaining $\mathbf{y}_{\mathbf{1 2}}^{(\mathbf{k})}$ via

$$
\begin{aligned}
\mathbf{y}_{\mathbf{1 2}} & =\left[\begin{array}{ll}
I_{n+n_{W}} & 0
\end{array}\right] \tilde{\mathbf{Y}}_{\mathbf{1 2}}\left[\begin{array}{c}
0_{(k-1) l \times l} \\
I_{l} \\
0_{(L-k-1) l \times l}
\end{array}\right] \\
& =\mathbf{A}_{\mathbf{0}} \mathbf{y}_{\mathbf{1 2}}^{(k-1)}-F^{*} \mathbf{P} \tilde{\mathbf{l}}^{(k)}(k=2, \ldots, L) .
\end{aligned}
$$

Let us finally consider the second line of (8) when the solution $\left(\mathbf{Y}_{\mathbf{2}}, \tilde{\mathbf{L}}\right)$ is applied. Using Lemma 1, we see that equivalently

$$
\begin{aligned}
-\mathbf{P} \tilde{\mathbf{L}}=D_{\perp}^{+} D_{W}\left[\begin{array}{lll}
\mathbf{B}_{\mathbf{0}}^{*} & D_{W}^{*}\left(D^{+}\right)^{*} & 0
\end{array}\right] \\
\\
+D_{\perp}^{+} \mathbf{C}_{\mathbf{0}}\left[\begin{array}{c}
\mathbf{A}_{\mathbf{0}} \mathbf{Y}_{\mathbf{1 1}} \\
D^{+} \mathbf{C}_{\mathbf{0}} \mathbf{Y}_{\mathbf{1 1}} \\
{\left[\begin{array}{lll}
I_{(L-1) l} & 0
\end{array}\right] \mathbf{Y}_{\mathbf{1 2}}^{*}}
\end{array}\right]^{*} .
\end{aligned}
$$

With (14), this gives us expressions for the $\mathbf{P} \tilde{\mathbf{l}}^{(\mathbf{k})}$. Plugging them into (18) and (19) results in the recursion from the proposition.

Proof (of Proposition 4): Let us first show that $\left(\mathbf{Y}_{\mathbf{2}}, \mathbf{L}\right)$ actually solves (8). By Lemma 1 and definition of $\hat{\mathbf{B}}$ we have

$$
\mathbf{L}_{\mathbf{2}}=-\mathbf{P}^{+} D_{\perp}^{+}\left(D_{W} \hat{\mathbf{B}}^{*}+\hat{\mathbf{C}} \mathbf{Y}_{\mathbf{2}} \hat{\mathbf{A}}^{*}\right) .
$$

Since $\left(\mathbf{Y}_{\mathbf{2}}, \tilde{\mathbf{L}}\right)$ solves the second line of $(8), \Gamma=\tilde{\mathbf{L}}_{\mathbf{2}}$ solves the equation $-\mathbf{P} \Gamma=D_{\perp}^{+}\left(D_{W} \hat{\mathbf{B}}^{*}+\hat{\mathbf{C}} \mathbf{Y}_{2} \hat{\mathbf{A}}^{*}\right)$. Then, the least squares property of the Moore-Penrose pseudoinverse implies that also $\Gamma=\mathbf{L}_{2}$ solves it. This shows $\mathbf{P L}_{2}=\mathbf{P L}_{2}$. Since by the proof of Proposition 3 we already know that $\mathbf{P L}_{1}=$ $\mathbf{P} \tilde{\mathbf{L}}_{\mathbf{1}}$, this implies $\mathbf{P L}=\mathbf{P} \tilde{\mathbf{L}}$. Therefore, the second line of (8) holds for $\left(\mathbf{Y}_{2}, \mathbf{L}\right)$ because it holds for $\left(\mathbf{Y}_{2}, \tilde{\mathbf{L}}\right)$. Using that $\mathbf{P}=$ $\mathbf{P P}^{+} \mathbf{P}$ by the properties of the Moore-Penrose pseudoinverse, $\mathbf{P}=\mathbf{P}^{*}$, and $\mathbf{P L}=\mathbf{P} \tilde{\mathbf{L}}$, we obtain

$$
\tilde{\mathbf{L}}_{i}^{*} \mathbf{P} \tilde{\mathbf{L}}_{j}=\tilde{\mathbf{L}}_{i}^{*} \mathbf{P}^{*} \mathbf{P}^{+} \mathbf{P} \tilde{\mathbf{L}}_{j}=\mathbf{L}_{i}^{*} \mathbf{P}^{*} \mathbf{P}^{+} \mathbf{P} \mathbf{L}_{j}=\mathbf{L}_{i}^{*} \mathbf{P} \mathbf{L}_{j}
$$

for all $i, j \in\{1,2\}$, which implies $\mathbf{L}^{*} \mathbf{P L}=\tilde{\mathbf{L}}^{*} \mathbf{P} \tilde{\mathbf{L}}$. Thus, also the first line of (8) holds for $\left(\mathbf{Y}_{\mathbf{2}}, \mathbf{L}\right)$. To see that $\left(\mathbf{Y}_{\mathbf{2}}, \mathbf{L}\right)$ is stabilizing, note that $\left(\mathbf{Y}_{\mathbf{1 1}}, \mathbf{L}_{\mathbf{1}}\right)$ is a stabilizing solution to (12), and apply (15).

Finally, the formula for $\mathbf{L}_{\mathbf{0}}$ follows directly from the fact that $\mathbf{P}=P$ (cf. the proof of Prop. 3) and Lemma 1.

\section{REFERENCES}

[1] K. Zhou, J. Doyle, and K. Glover, Robust and Optimal Control. Upper Saddle River, NJ: Prentice-Hall, 1996.

[2] M. Vetterli, "A theory of multirate filter banks," IEEE Trans. Acoust., Speech, Signal Process., vol. 35, pp. 356-372, Mar. 1987.

[3] P. P. Vaidyanathan, "Theory and design of m-channel maximally decimated quadrature mirror filters with arbitrary $\mathrm{m}$, having the perfect-reconstruction property," IEEE Trans. Acoust., Speech, Signal Process., vol. 35, pp. 476-492, Apr. 1987.

[4] P. P. Vaidyanathan, Multirate Systems and Filter Banks. Englewood Cliffs, NJ: Prentice-Hall, 1993.

[5] G. Gu and E. Badran, "Optimal design for channel equalization via the filterbank approach," IEEE Trans. Signal Process., vol. 52, no. 2, pp. 536-545, Feb. 2004

[6] L. Chai, J. Zhang, C. Zhang, and E. Mosca, "Optimal noise reduction in oversampled pr filter banks," IEEE Trans. Signal Process., vol. 57, no. 10 , pp. 3844-3857, Oct. 2009.

[7] A. Oppenheim, R. W. Schafer, and J. R. Buck, Discrete-Time Signal Processing, 2nd ed. Upper Saddle River, NJ: Prentice-Hall, 1999. 
[8] S. Wahls and H. Boche, "Realizable equalizers for frequency selective mimo channels with cochannel interference," presented at the IEEE Int. Conf. Acoustics, Speech, Signal Processing (ICASSP), Taipei, Taiwan, Apr. 2009.

[9] A. Saberi, A. A. Stoorvogel, and P. Sannuti, "Inverse filtering and deconvolution," Int. J. Robust Nonlinear Control, vol. 11, no. 2, pp. 131-156, 2001.

[10] L. Li and G. Gu, "Design of optimal zero-forcing precoders for mimo channels via optimal full information control," IEEE Trans. Signal Process., vol. 53, no. 8, pp. 3238-3246, Aug. 2005.

[11] G. Gu and L. Li, "Worst-case design for optimal channel equalization in filterbank transceivers," IEEE Trans. Signal Process., ser. 9, vol. 51, pp. 2424-2435, Sep. 2003.

[12] S. Wahls, H. Boche, and V. Pohl, "Zero-forcing precoding for frequency selective mimo channels with $h^{\infty}$ criterion and causality constraint," Signal Process., vol. 89, pp. 1754-1761, Sep. 2009.

[13] S. Kung, Y. Wu, and X. Zhang, "Bezout space-time precoders and equalizers for mimo channels," IEEE Trans. Signal Process., vol. 50, no. 10, pp. 2499-2514, Oct. 2002.

[14] A. Krishna and K. Hari, "Filter bank precoding for fir equalization in high-rate mimo communications," IEEE Trans. Signal Process., vol. 54 , no. 5, pp. $1645-1652,5,2006$.

[15] A. Varga, "Computing generalized inverse systems using matrix pencil methods," Int. J. Appl. Math. Comput. Sci., vol. 11, no. 5, pp. $1055-1068,2001$.

[16] L. Chai, J. Zhang, C. Zhang, and E. Mosca, "Frame-theory-based analysis and design of oversampled filter banks: Direct computational method," IEEE Trans. Signal Process., vol. 55, no. 2, pp. 507-519, Feb. 2007.

[17] L. Gan and C. Ling, "Computation of the para-pseudoinverse for oversampled filter banks: Forward and backward Greville formulas," IEEE Trans. Signal Process., vol. 56, pp. 5851-5860, Dec. 2008.

[18] H. Babic, S. K. Mitra, C. Creusere, and A. Das, "Perfect reconstruction recursive QMF banks for image subband coding," presented at the Asilomar Conf. Sig. Syst. Comp., Pacific Grove, CA, Nov. 1991.

[19] P. P. Vaidyanathan and T. Chen, "Role of anticausal inverses in multirate filter-banks-Part i: System-theoretic fundamentals," IEEE Trans. Signal Process., vol. 43, no. 5, pp. 1090-1102, May 1995.

[20] C. Oara, "Constructive solutions to spectral and inner-outer factorizations with respect to the disk," Automatica, vol. 41, pp. 1855-1866, Nov. 2005.

[21] K. Y. Polyakov, "Singular $\mathcal{H}_{2}$-optimization problems for discrete-time systems," Automat. Remote Control, vol. 66, pp. 356-368, Mar. 2005.

[22] V. Ionescu, C. Oara, and M. Weiss, Generalized Riccati Theory and Robust Control: A Popov Function Approach. Chichester, U.K.: Wiley, 1998.

[23] P. Vandewalle, J. Kovacevic, and M. Vetterli, "Reproducible research in signal processing - What, why, and how," IEEE Signal Process. Mag., vol. 26, pp. 37-47, May 2009.

[24] A. Scaglione, G. Giannakis, and S. Barbarossa, "Redundant filterbank precoders and equalizers-Part I: Unification and optimal designs," IEEE Trans. Signal Process., vol. 47, no. 7, pp. 1988-2006, Jul. 1999.
[25] H. L. Trentelman and A. A. Stoorvogel, "Sampled-data and discrete-time $h_{2}$ optimal control," SIAM J. Control Optim., vol. 33, pp. 834-862, May 1995.

[26] G. Golub and C. V. Loan, Matrix Computations, 3rd ed. Baltimore, MD: The Johns Hopkins Univ. Press, 1996.

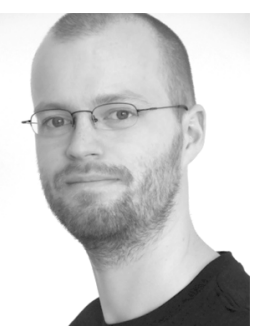

Sander Wahls (S'09) was born in Greifswald, Germany, in 1983. He received the Diplom-Mathematiker (Dipl.-Math.) degree from the Technische Universität Berlin, Germany, in 2007.

He is currently a Research Assistant at the same university, where he is also working towards the $\mathrm{Ph} . \mathrm{D}$. degree under the supervision of Prof. H. Boche. He is a Guest Researcher in the Broadband Mobile Communication Networks group at the Fraunhofer Institut für Nachrichtentechnik (HHI), Berlin, Germany. His research interests are in the areas of signal processing, automatic control, and communications.

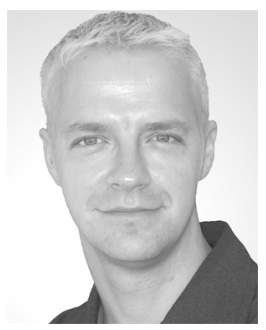

Holger Boche (M'04-SM'07) received the Dipl.-Ing. and Dr.-Ing. degrees in electrical engineering from the Technische Universität Dresden, Germany, in 1990 and 1994, respectively. He graduated in mathematics from the Technische Universität Dresden in 1992 and received the Dr.rer.nat. degree in pure mathematics from the Technische Universität Berlin, Germany, in 1998.

From 1994 to 1997, he did postgraduate studies in mathematics at the Friedrich-Schiller Universität Jena, Germany. In 1997, he joined the Heinrich-Hertz-Institut (HHI) für Nachrichtentechnik Berlin. Since 2002, he has been Full Professor for Mobile Communication Networks at the Technische Universität Berlin at the Institute for Communications Systems. In 2003, he became Director of the Fraunhofer German-Sino Lab for Mobile Communications, Berlin, Germany, and since 2004 he has also been Director of the Fraunhofer Institute for Telecommunications (HHI), Berlin, Germany. He was Visiting Professor at the ETH Zurich during winter term 2004 and 2006 and at KTH Stockholm during summer term 2005.

Prof. Boche received the Research Award "Technische Kommunikation" from the Alcatel SEL Foundation in October 2003, the Innovation Award from the Vodafone Foundation in June 2006, and the Gottfried Wilhelm Leibniz Prize from the Deutsche Forschungsgemeinschaft (German Research Foundation) in 2008. He was corecipient of the 2006 IEEE Signal Processing Society Best Paper Award and recipient of the 2007 IEEE Signal Processing Society Best Paper Award. He is a member of IEEE Signal Processing Society SPCOM and SPTM Technical Committee. He was elected a member of the German Academy of Sciences (Leopoldina) in 2008 and of Berlin Brandenburg Academy of Sciences and Humanities in 2009. 